\title{
Detection of serum antinuclear antibodies in lymphoma patients
}

\author{
H.Y. Zou ${ }^{1 *}$, X. Gu${ }^{2 *}$, W.Z. Yu' ${ }^{1}$ Z. Wang ${ }^{1}$ and M. Jiao ${ }^{1}$ \\ ${ }^{1}$ Institute of Clinical Medicine, Urumqi General Hospital of Lanzhou Military \\ Command Area, Urumqi, China \\ ${ }^{2}$ Department of Pathology, The First Affiliated Hospital of Guangzhou Medical College, \\ Guangzhou, China \\ *These authors contributed equally to this study. \\ Corresponding author: H.Y. Zou \\ E-mail: zouhongy2@126.com \\ Genet. Mol. Res. 14 (4): 16546-16552 (2015) \\ Received August 27, 2015 \\ Accepted October 24, 2015 \\ Published December 11, 2015 \\ DOI http://dx.doi.org/10.4238/2015.December.11.1
}

ABSTRACT. We investigated the presence of serum antinuclear antibodies (ANAs) and autoantibodies and their relationship with serum prognostic indicators in lymphoma patients. The study population comprised 127 patients diagnosed with lymphoma and 138 healthy control subjects. The blood samples of the participants were assayed for ANAs by immunofluorescence, and autoantibodies were detected by western blotting. Serum ANAs were detected in 31.5 (40/127) and 6.5\% (9/138) of lymphoma patients and control subjects, respectively. There was a statistically significant difference between the lymphoma and the control groups $(P<0.05)$. The level of lactate dehydrogenase in the ANA-positive subjects was significantly lower than in the ANA-negative subjects $(P<$ $0.05)$. Low ANA titers (1:100) were commonly found in the ANA-positive subjects and the control subjects, and the fluorescence models were diverse. Autoantibodies were found in 35\% (14/40) of the ANA-positive patients by western blotting. Detection of ANAs in lymphoma patients helps in determining the diagnosis and prognosis of lymphoma, but has 
no independent diagnostic value; there are still various autoantibodies of unknown significance that require further study.

Key words: Antinuclear antibodies; Autoantibodies; Lymphoma

\section{INTRODUCTION}

Antinuclear antibodies (ANAs) are a group of autoantibodies that target antigens of the nucleus in mammals. They are important serological markers of autoimmune disease. In recent years, increasing attention has been paid to the relationship between tumors and autoimmunity. Researchers have shown that ANAs relate not only to autoimmune diseases, but also to the majority of cancers, suggesting that they may be involved in the pathological processes of cancers and other diseases (Ma et al., 2010; Li et al., 2011). ANAs and autoantibodies in lymphoma patients were detected by immunofluorescence and western blotting to explore the clinical significance of ANAs in lymphoma.

\section{MATERIAL AND METHODS}

\section{Subjects}

Serum samples were collected from 127 patients diagnosed with malignant lymphoma (75 males and 52 females) in the First and Second Affiliated Hospitals of Xinjiang Medical University between 2005 and 2010. The study population comprised 86 Han patients, 36 Uygur patients, and 5 Kazakh patients, aged 6-87 years and with a median age of 56 years. According to the 2008 World Health Organization classification criteria for tumors of the hematopoietic and lymphoid system, the subjects comprised 14 cases of Hodgkin's lymphoma (HL) and 113 cases of non-Hodgkin's lymphoma (NHL) (including 14 cases of T-cell lymphoma and 99 cases of B-cell lymphoma). Healthy control subjects (138) without autoimmune diseases and of a similar age and gender to the patients group were also selected.

\section{Methods}

Venous blood $(2 \mathrm{~mL})$ was collected from each subject, and the serum was separated by centrifugation at $2500 \mathrm{~g}$, for $10 \mathrm{~min}$ after natural coagulation. The serum was stored at $-70^{\circ} \mathrm{C}$. Serum ANA titer levels were detected by indirect immunofluorescence using Hep-2 (epithelial cell lines of human throat cancer) and monkey liver tissue sections as substrates, and fluorescein isothiocyanate (FITC)-labeled goat anti-human IgG as secondary fluorescent antibodies, with a serum dilution of 1:100. A serum ANA-positive result was defined as the presence of specific green fluorescence in tissues or cells at a serum dilution of $\geq 1: 100$.

\section{Western blotting}

ANA-positive sera obtained by indirect immunofluorescence assay were used in the detection of autoantibodies by western blotting. Fifteen autoantibodies were detected: anti-snRNP/ Sm, anti-Sm, anti-SSA, anti-SSB, anti-dsDNA, anti-histone, anti-ribosomal P protein, anti-Scl-70, anti-Jo-1, anti-Ro-52, anti-PCNA (anti-proliferating cell nuclear antigen antibody), anti-ACA (anti- 
centromere antibody), anti-PM-Scl, anti-M2, and anti-nucleosome antibodies.

All the tests described above were conducted using a German EUROIMMUN kit (Berlin, German) in strict accordance with the manufacturer instructions for operation and interpretation of the experimental results.

\section{Statistics}

The count data are described by the number or percentage of cases. PEMS 3.1 statistical software was used to compare the rates using $x^{2}$ analysis.

\section{RESULTS}

\section{Relationship between ANA assay and lymphoma}

Serum ANAs were detected in 31.5\% (40/127) and 6.5\% (9/138) of lymphoma patients and control subjects, respectively. There was a statistically significant difference between the positive rates of the lymphoma group and the control group $(P<0.05)$, but no significant difference between the $\mathrm{HL}$ and NHL groups, or the T-cell lymphoma and B-cell lymphoma groups $(P>0.05)$. There was no statistically significant difference in age, sex, or nationality $(P>0.05)$. The results are given in Table 1.

\begin{tabular}{|c|c|c|c|}
\hline Group & $\mathrm{N}$ & No. of ANA-positive & Positive rate (\%) \\
\hline Control & 138 & 9 & 6.5 \\
\hline Lymphoma & 127 & 40 & 31.5 \\
\hline $\mathrm{HL}$ & 14 & 5 & 35.7 \\
\hline $\mathrm{NHL}$ & 113 & 35 & 30.9 \\
\hline T-cell lymphoma & 14 & 3 & 21.4 \\
\hline B-cell lymphoma & 99 & 32 & 32.3 \\
\hline \multicolumn{4}{|l|}{ Age } \\
\hline$\leq 50$ & 52 & 15 & 28.8 \\
\hline$>50$ & 75 & 25 & 33.3 \\
\hline \multicolumn{4}{|l|}{ Gender } \\
\hline Male & 75 & 25 & 33.3 \\
\hline Female & 52 & 15 & 28.8 \\
\hline \multicolumn{4}{|l|}{ Nationality } \\
\hline Han & 86 & 30 & 34.9 \\
\hline Uygur & 36 & 9 & 25.0 \\
\hline Kazak & 5 & 1 & 20.0 \\
\hline
\end{tabular}

ANA = antinuclear antibody; HL = Hodgkin's lymphoma; NHL = non-Hodgkin's lymphoma. There were statistically significant differences between the ANA-positive rates in the lymphoma group and the control group.

The level of LDH in the ANA-positive subjects was significantly lower than in the ANA-negative subjects $(P<0.05)$, but there was no significant difference in the serum levels of $\beta 2$-microglobulin $(\beta-2 M G)$ and C-reactive protein $(C R P)$ between the two groups of subjects $(P>0.05)$ (Table 2$)$.

\section{ANA titer analysis and fluorescence models}

ANA titer analysis showed that low ANA titers (1:100) were commonly found in ANApositive subjects, accounting for $75 \%$ (30/40); titers of $1: 320$ accounted for $17.5 \%$ ( $7 / 40)$; and titers 
of $1: 1000$ accounted for $7.5 \%$ (3/40). In the nine ANA-positive cases of the control group, there were seven cases with a titer of 1:100, one case with a titer of 1:320, and one case with a titer of 1:1000.

Further analysis of the ANA fluorescence models showed that the ANA fluorescence models of lymphoma patients were diversified, and nine different models were detected, of which the nucleolus model, the nuclear homogeneous model, and the nuclear particle model were the most common. Four kinds of fluorescence models were detected in the ANA-positive subjects in the control group, as shown in Table 3.

\begin{tabular}{|c|c|c|c|c|}
\hline Group & $\mathrm{N}$ & $x \pm s$ & $t$-test & $P$ value \\
\hline \multicolumn{5}{|l|}{ LDH } \\
\hline $\mathrm{ANA}(+)$ & 38 & $210.9316 \pm 83.9300$ & 2.1282 & $P<0.05$ \\
\hline ANA(-) & 77 & $297.4805 \pm 336.2700$ & & \\
\hline \multicolumn{5}{|l|}{$\beta-2 M G$} \\
\hline $\mathrm{ANA}(+)$ & 27 & $4.8237 \pm 5.6773$ & 0.2865 & $P>0.05$ \\
\hline ANA(-) & 48 & $4.4163 \pm 6.3055$ & & \\
\hline \multicolumn{5}{|l|}{ CRP } \\
\hline ANA(+) & 22 & $28.9105 \pm 41.7631$ & 0.6586 & $P>0.05$ \\
\hline ANA (-) & 40 & $22.421 \pm 26.6877$ & & \\
\hline
\end{tabular}

ANA = antinuclear antibody; $\mathrm{LDH}=$ lactate dehydrogenase; $\beta-2 \mathrm{MG}=\beta 2$-microglobulin; $\mathrm{CRP}=\mathrm{C}$-reactive protein.

Table 3. Comparison of ANA fluorescence models in the different groupsANA fluorescence models.

\begin{tabular}{lcc}
\hline & Control $(\mathrm{N}=138)$ & Lymphoma $(\mathrm{N}=127)$ \\
\hline Nucleolus & 5 & 14 \\
Nuclear dots & 1 & 0 \\
Nuclear homogeneous & 0 & 9 \\
Nuclear particle & 2 & 9 \\
Cytoplasmic fiber & 1 & 1 \\
Spindle & 0 & 4 \\
Centrioles & 0 & 3 \\
Cytoplasmic granules & 0 & 6 \\
Golgi apparatus & 0 & 3 \\
\hline
\end{tabular}

ANA = antinuclear antibody.

Among the 40 cases of ANA-positive lymphoma subjects there were 32 cases with a single fluorescent model. Another eight cases had mixed-type fluorescent models; there were three cases of nuclear homogeneous and nuclear particle models, one case of nucleolar and cytoplasmic granules, one case of nucleolar and spindle, one case of nuclear particle and centrioles, one case of Golgi apparatus and spindle, and one case of nuclear particle, centrioles and cytoplasmic fiber.

\section{Autoantibody spectrum assay}

Forty serum samples of ANA-positive cases were further evaluated by western blotting. Serum autoantibodies were detected in 35\% (14/40) of lymphoma patients and control subjects, with several positive bands (Table 4). Seven kinds of autoantibodies were found in the lymphoma patients, while only three kinds of autoantibodies were discovered in the control group. 


Table 4. Western blotting of ANA-positive serum.
\begin{tabular}{lccccccc}
\hline Groups & SSA & SSB & Ro-52 & M2 & PM & Ds-DNA & Scl-70 \\
\hline Lymphoma & 3 & 1 & 8 & 3 & 1 & 1 & 1 \\
Control & 1 & 0 & 1 & 0 & 0 & 0 & 0 \\
\hline
\end{tabular}

ANA = antinuclear antibody.

\section{DISCUSSION}

Our results showed that in patients with malignant lymphoma, the ANA-positive rate was $31.5 \%$ - significantly higher than in the control group $(6.5 \%, \mathrm{P}<0.05)$. Lymphoma often occurs in people with immune dysfunction. Some normal individuals also have autoantibodies, and the rate of occurrence increases with age (Li et al., 2011). Furthermore, tumor incidence is positively correlated to age. Therefore, it is still uncertain whether cancer patients with autoantibodies reflect cancerrelevance or age-relevance. However, the ANA-positive rate in our study was not related to age.

Research has shown that the immunogenicity of tumor cells can induce the production of a variety of autoantibodies: with SP2/0 tumor cell nuclei and normal DNA-immunized Balb/c mice, anti-dsDNA, anti-histone, anti-Sm, anti-ds-DNA, anti-ss-DNA, anti-histone, anti-Sm, antiSS-A, and anti-SS-B were obtained (Shen et al.,2011). The experimental results showed that ANAs have anti-tumor activity. This may be mediated by antibody-dependent cell-mediated cytotoxicity (ADCC), by cytokines that enhance the immune function induced by ANA immune complexes, and by eliminating or reducing the inhibitory effect of extracellular chromatin on natural killer (NK) cell activity through the binding of ANAs and extracellular nuclear chromatin released from apoptotic tumor cells (Lu et al., 1998). Some cancer patients have been misdiagnosed in clinical situations owing to various serum autoantibodies and manifestations of rheumatism (Abu-Shakra et al., 2001; Solans-Laqué et al., 2004; Mu et al., 2006).

It has been reported that the mortality rate of cancer patients with autoimmune diseases is significantly lower than that of general cancer patients; the presence of circulating ANAs in lung cancer and colon cancer patients is related to prolonged disease progression and improved survival rate (Erkanli et al., 2006), but there are also contrary reports (Altintas et al., 2008). Autoimmune response is common in patients with lung cancer, and some asymptomatic patients may present with high levels of autoantibodies before the discovery of solid tumors or symptoms (Zhong et al., 2006; Murray et al., 2010).

The LDH level is a clinical prognosis indicator for patients with lymphoma, and high levels commonly correspond to poor prognosis (Shen and Wang, 2010). In this study, the ANA-negative subjects had higher LDH levels, suggesting that the prognosis for the ANA-negative subjects would be worse than for the positive subjects. This was consistent with the reported findings that ANA-positive patients with lung cancer and colon cancer have a prolonged disease progression and improved survival rate (Erkanli et al., 2006). This may be owing to the anti-tumor activity of ANAs mediating ADCC in vivo, the effects of cytokines, and the elimination or reduction of the inhibitory effect of extracellular chromatin on NK cell activity, as described above, as well as to other factors (Lu et al., 1998).

The results also showed that the ANA fluorescent models of the patients with malignant lymphoma were more diverse and abundant than those of the control group, and the spectrum of autoantibodies in the patient group was relatively wide. ANA titer analysis showed that ANApositive lymphoma patients commonly had low titers (1:100), accounting for $75 \%$, with few high titers. Our findings are consistent with previously reported results of ANAs in malignancies (Yang 
et al., 2005), indicating that malignant lymphoma patients are subject to autoimmune phenomena, and such immune abnormalities and autoantibody overexpression may be another characteristic of lymphoma. Autoantibodies were detected in the sera of patients with lymphoma in the nucleolus, nuclear matter, cytoplasm, and many other sites. Autoantibodies were commonly detected in the nucleolus model (14 cases), the nuclear particle model ( 9 cases), and the nuclear homogeneous model ( 9 cases), suggesting that the targeted antigens for autoantibodies in lymphoma patients were mostly nucleolar antigens, ribonucleoproteins, histones, polynucleotides, and other proteins related to biosynthesis and cell proliferation. This study suggests that autoantibody detection will not only contribute to the diagnosis of patients with autoimmune diseases and healthy individuals, but also to the diagnosis of patients with lymphoma. Unlike previous domestic reports, in this study, mitosis-associated antigen antibodies were detected (spindle and central grain), and antibodies were also found in the Golgi apparatus. The clinical significance and diagnostic value of this discovery are unclear and require further research.

There were no significant difference between the ANA-positive rates in the $\mathrm{HL}$ and $\mathrm{NHL}$ groups, or between the T-cell lymphoma and B-cell lymphoma groups. Therefore, the detection of ANAs and the autoantibody spectrum contributes little to the diagnosis of different types of lymphoma, and needs to be combined with other clinical and laboratory markers.

In this study, $87(68.5 \%)$ of the 127 lymphoma patients were found to be ANA-negative, and among the 40 ANA-positive patients, autoantibodies were detected in only 14 cases, suggesting that there may be other autoantibodies in lymphoma patients besides the common autoantibodies associated with autoimmune disease. This indicates that tumor cells may produce other antibodies besides ANAs and common autoantibodies through epitope expansion. Therefore, further research into the nature and biological effects of these autoantibodies will have a profound influence on the indepth understanding of autoimmune lymphoma, as well as on its diagnosis, treatment, and prognosis.

\section{Conflicts of interest}

The authors declare no conflict of interest.

\section{ACKNOWLEDGMENT}

Research supported by the National Natural Science Foundation of China (\#81172840 and \#30860096).

\section{REFERENCES}

Abu-Shakra M, Buskila D, Ehrenfeld M, Conrad K, et al. (2001). Cancer and autoimmunity: autoimmune and rheumatic features in patients with malignancies. Ann. Rheum. Dis. 60: 433-441.

Altintas A, Cil T, Pasa S, Danis R, et al. (2008). Clinical significance of elevated antinuclear antibody test in patients with Hodgkin's and Non-Hodgkin's lymphoma: a single center experience. Minerva Med. 99: 7-14.

Erkanli A, Taylor DD, Dean D, Eksir F, et al. (2006). Application of Bayesian modeling of autologous antibody responses against ovarian tumor-associated antigens to cancer detection. Cancer Res. 66: 1792-1798.

Li QZ, Karp DR, Quan J, Branch VK, et al. (2011). Risk factors for ANA positivity in healthy persons. Arthritis Res. Ther. 13 : R38.

Lu L, Li J, Wang M and Wu H (1998). Induction of anti-DNA antibodies by immunization with activated lymphocytes and active chromatin. Chin. Med. J. (Engl). 111: 524-526.

Ma XY, Zhang H and Qu YJ (2010). Autoantibodies and the correlation of malignant tumor research. Ji Lin Yi Xue. 31: 4801-4802. Mu R, Li WY and Su ZG (2006). Lung cancer patients with high drop degree antinuclear antibodies and anti SSA. Zhong Hua 
Nei Ke Za Zhi. 45: 766

Murray A, Chapman CJ, Healey G, Peek LJ, et al. (2010). Technical validation of an autoantibody test for lung cancer. Ann. Oncol. 21: 1687-1693.

Shen J and Wang KT (2010). Clinical significance of serum LDH in non-hodgkin's lymphoma. Zhong Guo Yi Yao Zhi Nan. 8: 116-117.

Shen LL, Han XH and Zheng XJ (2011). With SP2/0 tumor cell nucleus or DNA immunization with induction of antinuclear antibodies in mice. Shang Hai Mian Yi Xue Za Zhi. 21: 219-220.

Solans-Laqué R, Pérez-Bocanegra C, Salud-Salvia A, Fonollosa-Plá V, et al. (2004). Clinical significance of antinuclear antibodies in malignant diseases: association with rheumatic and connective tissue paraneoplastic syndromes. Lupus 13: 159-164.

Yang CG, Wu L and Wang MY (2005). Cancer patients to explore the clinical significance of the serum autoantibodies. Guo Wai Yi Xue Lin Chuang Sheng Wu Hua Xue Fen Ce. 26: 942-943.

Zhong L, Coe SP, Stromberg AJ, Khattar NH, et al. (2006). Profiling tumor-associated antibodies for early detection of nonsmall cell lung cancer. J. Thorac. Oncol. 1: 513-519. 